\title{
A Case of Neuromyelitis Optica Masquerading as Miller Fisher Syndrome
}

\author{
Yuka Furutani $^{\mathrm{a}} \quad$ Masayuki Hata $^{\mathrm{a}} \quad$ Kazuaki Miyamoto $^{\mathrm{a}}$ Yusaku Moribata $^{\mathrm{b}}$ \\ Nagahisa Yoshimura ${ }^{a}$ \\ Departments of ${ }^{\mathrm{a}}$ Ophthalmology and Visual Sciences and ${ }^{\mathrm{b}}$ Diagnostic Imaging and \\ Nuclear Medicine, Kyoto University Graduate School of Medicine, Kyoto, Japan
}

\section{Key Words}

Neuromyelitis optica · Abduction palsy · Optic neuritis · Miller Fisher syndrome

\begin{abstract}
A 22-year-old woman presented with double vision that she had experienced since an infection 2 weeks previously. A neurological examination showed limited bilateral eye abduction, mimicking Miller Fisher syndrome. However, T2-weighted magnetic resonance imaging of her brain revealed hyperintense areas in the tegmentum of the pons, including the abducens nucleus, and her serum anti-aquaporin-4 antibody test was positive. She was finally diagnosed with neuromyelitis optica. Intravenous high-dose steroid therapy immediately improved the patient's abduction palsy, but bilateral optic neuritis manifested during the treatment. Subsequent treatment with plasma exchange improved her optic neuritis symptoms.

(C) 2014 S. Karger AG, Basel
\end{abstract}

\section{Introduction}

Neuromyelitis optica (NMO) is a severe autoimmune inflammatory demyelinating disease of the central nervous system (CNS) that preferentially affects the optic nerve and spinal cord [1]. Compared with typical optic neuritis, NMO is often not improved by intravenous steroid therapy, which is the standard treatment for refractory or severe optic neuritis, and can result in severe visual impairment $[2,3]$.

Brainstem involvement has recently been described in NMO, and the predominant manifestations are vomiting and hiccups, which mainly occur at disease onset [4]. In

Masayuki Hata, MD

Department of Ophthalmology and Visual Sciences

Kyoto University Graduate School of Medicine

54 Shogoin Kawahara-cho, Sakyo-ku, Kyoto 606-8507 (Japan)

E-Mail trj74h6@kuhp.kyoto-u.ac.jp 
Furutani et al.: A Case of Neuromyelitis Optica Masquerading as Miller Fisher Syndrome

addition, some patients with NMO experience diplopia with external ophthalmoplegia $[3,5$, 6]. However, there are no reports describing NMO with abduction palsy.

Here we describe a novel case of NMO with bilateral abduction palsy following an infection, the symptoms of which were initially suggestive of Miller Fisher syndrome. This case also brings forth the differences in the efficacy of steroids for treating lesions of the CNS and brainstem in NMO.

\section{Case Report}

A 22-year-old woman presented at our hospital complaining of double vision that had lasted 2 weeks. One month prior to the visit, she had shown symptoms such as fever and a cough and had been diagnosed with upper tract inflammation, possibly due to a viral infection. Two weeks later, she had experienced double vision, vomiting, and hiccups.

On examination, her corrected visual acuity was found to be $1.5 \mathrm{OU}$, and her light reflex was complete and prompt. There was no relative afferent pupillary defect (RAPD). She showed esotropia of 25 prism diopters in the primary position with restricted eye movement to both the right and left eye with right dominance (fig. 1a). Visual field assessment, fundoscopy, and the Ishihara color blindness tests showed no abnormality. Systemic neurological examinations showed hyperreflexia in both the upper and lower extremities, but no signs of motor ataxia.

Based on these findings, Miller Fisher syndrome was initially suspected. To rule out other diseases, brain magnetic resonance imaging (MRI) was performed. T2-weighted and fluid-attenuated inversion recovery images showed hyperintense areas in the tegmentum of the pons, including the abducens nucleus with right dominance, the dorsal medulla oblongata including the area postrema, the left ventral midbrain, the periphery of the lateral and third ventricular regions, and the left middle cerebellar peduncle (fig. 1b). A spinal MRI revealed a longitudinal lesion extending from the cervical to the thoracic spinal cord (fig. 1b). There was no finding suggesting optic nerve inflammation or lesions of the pontocerebellar cistern, clivus, or cavernous sinus that could have directly damaged the oculomotor or abducens nerves. The patient's cerebrospinal fluid was hypercellular ( $41 \mathrm{cells} / \mu \mathrm{l}$, $95.1 \%$ mononuclear cells) with elevated levels of total protein $(75.5 \mathrm{mg} / \mathrm{dl})$; however, an oligoclonal band was not detected. A test for the anti-aquaporin-4 (AQP4) antibody in the patient's peripheral blood was positive, and she was diagnosed with NMO based on the established criteria [7]. According to the clinical features of this patient, acute disseminated encephalomyelitis could be considered. However, we excluded this based on the patient's relapsing clinical course and positive reaction of the anti-AQP4 antibody. In addition, antinuclear antibody and other autoantibodies were all negative, and this patient had no abnormalities in the anterior portion, optic media, or fundus of the eye and no cutaneous or joint symptoms suggesting other autoimmune diseases such as systemic lupus, Sjögren's syndrome, or sarcoidosis.

The patient was initially treated with intravenous methylprednisolone therapy (IVMP; $1,000 \mathrm{mg} /$ day for 3 days) followed by oral prednisolone ( $40 \mathrm{mg}$ /day for 7 days, with gradual tapering). After IVMP, her eye movement gradually improved, and symptoms of diplopia, nausea, and hiccupping disappeared. The hyperintense lesions on the tegmentum of the pons also diminished (fig. 2a).

A week later, she experienced visual field loss and pain during left eye movement. An ophthalmologic examination revealed RAPD, decreased critical flicker frequency (CFF), and an upper visual field defect in the left eye (fig. $2 \mathrm{~b}$ ). Tests for eye position and eye movement 
Furutani et al.: A Case of Neuromyelitis Optica Masquerading as Miller Fisher Syndrome

and a fundoscopy were normal. Around the same time, she complained of left arm and right leg paresis and a left-sided headache. Contrast-enhanced MRI showed swelling and enhancement of the bilateral optic nerves, suggesting optic neuritis (fig. 2c).

Although several IVMP treatments were administered again, the patient's optic neuritis did not show any improvements. We considered that she was at a high risk of relapse, and plasma exchange was performed five times. Two weeks later, the defects in her visual field and CFF had almost normalized (fig. 2b).

\section{Discussion}

The course of this patient highlights three important clinical findings. NMO can present as an abduction defect, and infection can precede the neurological symptoms, mimicking Miller Fisher syndrome. The response of the lesions in the optic nerve and brainstem to IVMP during an acute NMO attack also differed.

First, the primary symptom of NMO can be eye movement disturbance. NMO is characterized by the presence of serum anti-AQP4 antibody, which targets AQP4 in astrocytic foot processes in the CNS and ependymal cells around the brainstem $[8,9]$. Previous reports on the characteristics of brainstem symptoms of NMO have shown that $17.3 \%$ of NMO patients with anti-AQP4 antibody positivity have oculomotor dysfunction [3]. However, the patterns of eye movement disturbance and the associated lesions were not analyzed in detail. Notably, there are no reports of patients with abduction defects. The present case exhibited a bilateral abduction deficit that was right dominant, which is consistent with the causative lesion in the abducens nucleus revealed by MRI findings. In addition, a previous study showed that diencephalon and brainstem MRI findings were predominant at an early onset in NMO patients [3]. In fact, the present patient presented with abduction palsy before her optic neuritis occurred. The different onset timing may be due to anatomical differences; the area postrema is not protected by the blood-brain barrier (BBB) [3].

Second, there are different efficacies for IVMP depending on whether the lesions are in the brainstem or optic nerve during acute attacks of NMO. According to pathological findings in NMO spinal lesions, astrocytes are first damaged by anti-AQP4 antibodies and complement proteins following an inflammatory demyelination event [7, 10]. As for optic nerve lesions due to NMO, no postmortem histological examinations have been reported. Based on experimental studies using animal models, it has been shown that optic nerves also exhibit decreased levels of AQP4 and glial fibrillary acidic protein, which is followed by the loss of optic nerve fibers and inflammatory cell infiltration [11]. However, the different types of edema regulated by AQP4 channels in the optic nerve and spinal cord suggest that the physiological function of the BBB might differ from that of the spinal cord [12]. In the optic nerve, necrotic changes might occur due to cytotoxic edema, resulting in permanent impairment. In the present case, intravenous steroid therapy improved the patient's abduction defect but did not halt the progression of her optic neuritis. Anatomical differences involving the BBB might be an explanation for this disparity. Moreover, there may be pathophysiological differences between CNS and brainstem lesions.

Plasma exchange finally ameliorated the optic neuritis, and the MRI evidence of brainstem lesions also indicated improvement. When no significant clinical improvement is observed following steroid treatment, plasma exchange has been shown to be effective for treating acute NMO [2]. Plasma exchange can suppress CNS inflammation by removing antiAQP4 antibodies [13]. Further studies are needed to determine whether plasma exchange could prevent the development of CNS lesions in the setting of limited brainstem lesions. 
Furutani et al.: A Case of Neuromyelitis Optica Masquerading as Miller Fisher Syndrome

Third, there is a possible association between the development of NMO and acute viral infection, which might activate the immune system and initiate autoimmunity. We first suspected the patient to have Miller Fisher syndrome based on her abduction defect after infection, although some cases of NMO following infection have been reported [14, 15].

These cases raise two possibilities. One is that, in some NMO patients, viral infections increase BBB permeability, which allows the autoantibody to enter the brain, although we have no data regarding leakage from the peripheral circulation to the patient's cerebrospinal fluid. Secondly, viral infection can trigger NMO-associated autoimmunity. In general, viruses induce autoimmunity through molecular mimicry and adjuvant-like activity during disease initiation, or through bystander activation with or without antigen spreading. Therefore, we could hypothesize that acute viral infection activates the immune system to initiate NMO.

In conclusion, NMO can cause brainstem lesions after an infection, and the symptoms can mimic those of Miller Fisher syndrome. In addition, optic neuritis can develop even if brainstem lesions are successfully controlled with steroid therapy. Plasma exchange may be needed to prevent the development of CNS lesions.

\section{Disclosure Statement}

The authors report no conflicts of interest.

\section{References}

1 Wingerchuk DM, Hogancamp WF, O’Brien PC, Weinshenker BG: The clinical course of neuromyelitis optica (Devic's syndrome). Neurology 1999;53:1107-1114.

2 Watanabe S, Nakashima I, Misu T, Miyazawa I, Shiga Y, Fujihara K, Itoyama Y: Therapeutic efficacy of plasma exchange in NMO-IgG-positive patients with neuromyelitis optica. Mult Scler 2007;13:128-132.

-3 Kremer L, Mealy M, Jacob A, Nakashima I, Cabre P, Bigi S, Paul F, Jarius S, Aktas O, Elsone L, Mutch K, Levy M, Takai Y, Collongues N, Banwell B, Fujihara K, de Seze J: Brainstem manifestations in neuromyelitis optica: a multicenter study of 258 patients. Mult Scler DOI: 10.1177/1352458513507822.

4 Misu T, Fujihara K, Nakashima I, Sato S, Itoyama Y: Intractable hiccup and nausea with periaqueductal lesions in neuromyelitis optica. Neurology 2005;65:1479-1482.

5 Shinoda K, Matsushita T, Furuta K, Isobe N, Yonekawa T, Ohyagi Y, Kira J: Wall-eyed bilateral internuclear ophthalmoplegia (WEBINO) syndrome in a patient with neuromyelitis optica spectrum disorder and antiaquaporin-4 antibody. Mult Scler 2011;17:885-887.

6 Garcia-Martin E, Pinilla I, Pueyo V, Gil L, Martinez-Morales J, Fernandez J: Bilateral internuclear ophthalmoplegia in a patient with Devic's neuromyelitis optica. Case Rep Neurol 2010;2:139-144.

7 Wingerchuk DM, Lennon VA, Lucchinetti CF, Pittock SJ, Weinshenker BG: The spectrum of neuromyelitis optica. Lancet Neurol 2007;6:805-815.

-8 Nagelhus EA, Veruki ML, Torp R, Haug FM, Laake JH, Nielsen S, Agre P, Ottersen OP: Aquaporin-4 water channel protein in the rat retina and optic nerve: polarized expression in Muller cells and fibrous astrocytes. J Neurosci 1998;18:2506-2519.

-9 Venero JL, Vizuete ML, Ilundain AA, Machado A, Echevarria M, Cano J: Detailed localization of aquaporin-4 messenger RNA in the CNS: preferential expression in periventricular organs. Neuroscience 1999;94:239250.

10 Lucchinetti CF, Guo Y, Popescu BF, Fujihara K, Itoyama Y, Misu T: The pathology of an autoimmune astrocytopathy: lessons learned from neuromyelitis optica. Brain Pathol 2014;24:83-97.

11 Matsumoto Y, Kanamori A, Nakamura M, Takahashi T, Nakashima I, Negi A: Sera from patients with seropositive neuromyelitis optica spectral disorders caused the degeneration of rodent optic nerve. Exp Eye Res 2014;119:61-69.

12 Saadoun S, Papadopoulos MC: Aquaporin-4 in brain and spinal cord oedema. Neuroscience 2010;168:10361046.

13 Kim KN, Jeoung JW, Park KH, Lee DS, Kim DM: Effect of lateral decubitus position on intraocular pressure in glaucoma patients with asymmetric visual field loss. Ophthalmology 2013;120:731-735.

14 Koga M, Takahashi T, Kawai M, Fujihara K, Kanda T: A serological analysis of viral and bacterial infections associated with neuromyelitis optica. J Neurol Sci 2011;300:19-22. 
Furutani et al.: A Case of Neuromyelitis Optica Masquerading as Miller Fisher Syndrome

15 Nishiyama S, Ito T, Misu T, Takahashi T, Kikuchi A, Suzuki N, Jin K, Aoki M, Fujihara K, Itoyama Y: A case of NMO seropositive for aquaporin-4 antibody more than 10 years before onset. Neurology 2009;72:19601961.

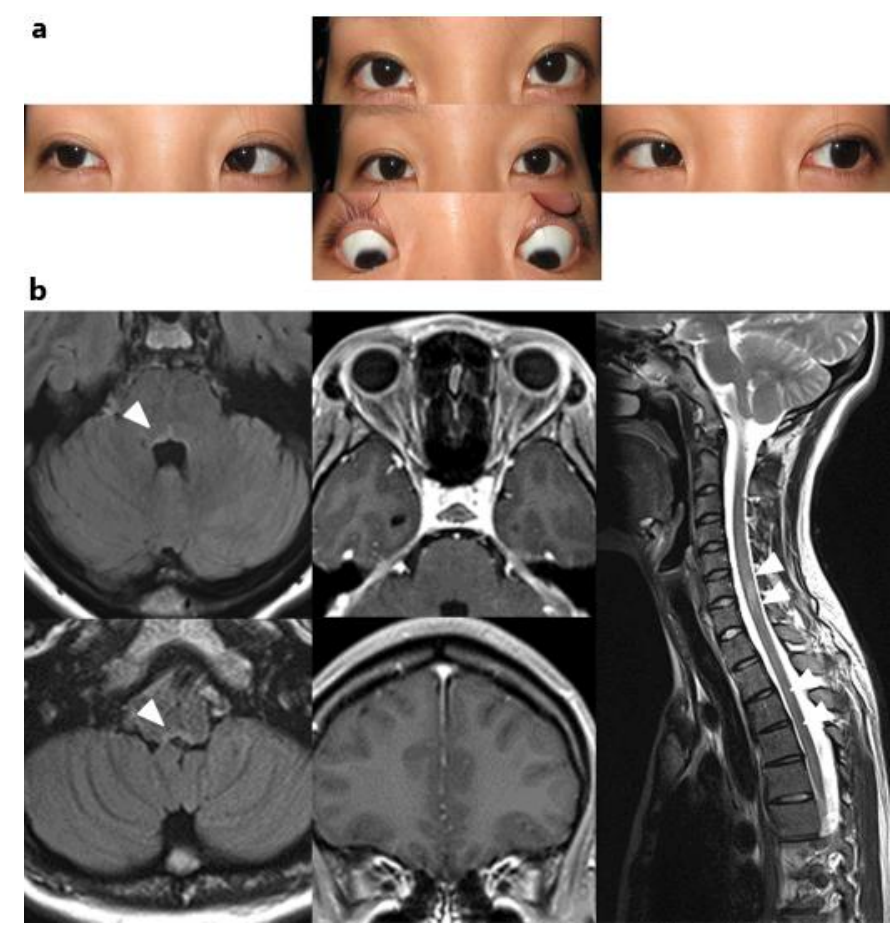

Fig. 1. a Eye movement test profile showing bilateral abduction deficit with right dominance. At presentation, the patient had esotropia in the primary position. b Axial fluid-attenuated inversion recovery MRI showing hyperintense areas (left panel) in the tegmentum of the pons with right dominance (top left) and the dorsal medulla oblongata including the area postrema (bottom left). No significant changes were noted in the optic nerves (middle panel). A sagittal spinal T2-weighted MRI revealed a longitudinal lesion (right panel). 
Furutani et al.: A Case of Neuromyelitis Optica Masquerading as Miller Fisher Syndrome
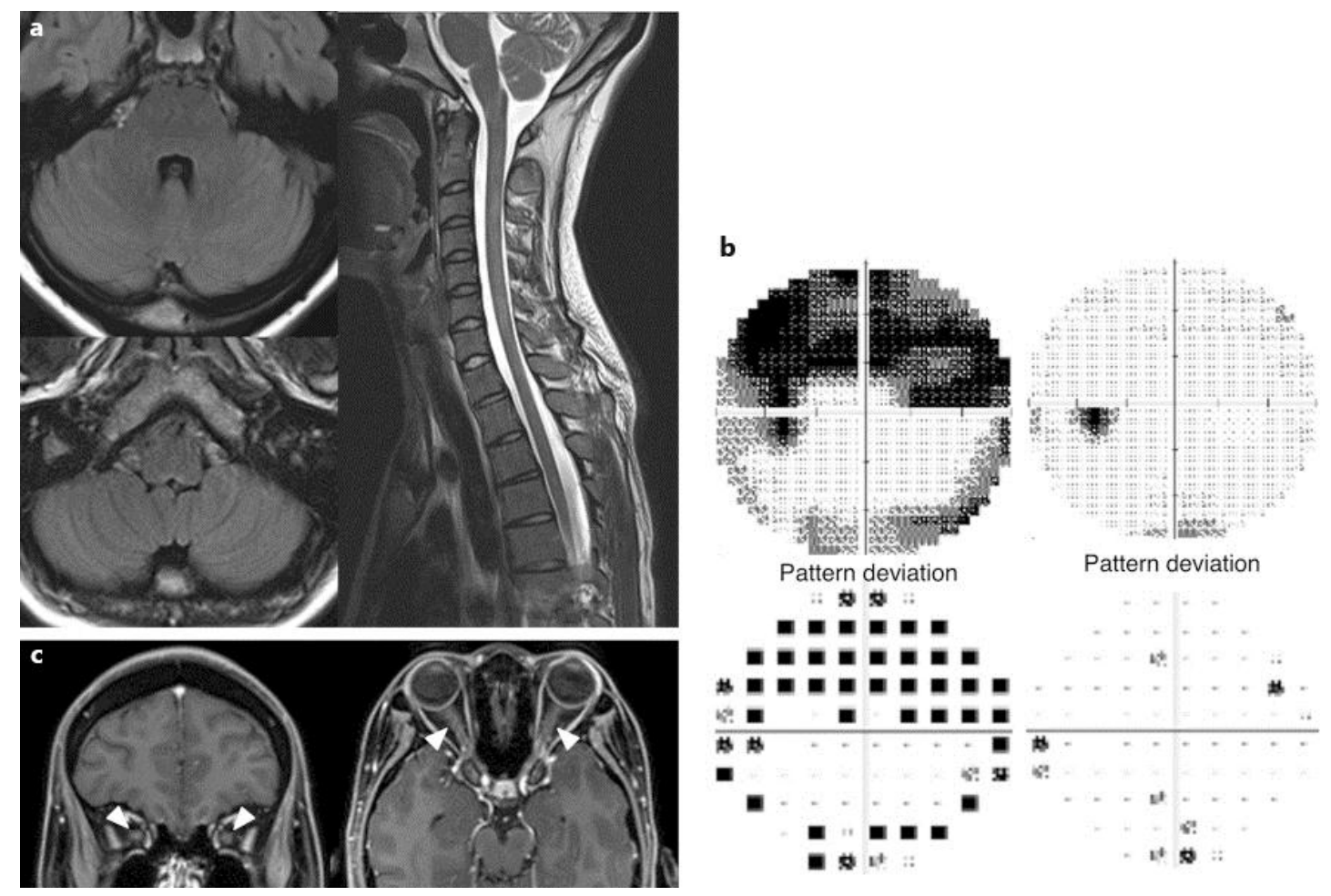

Fig. 2. a Images of axial fluid-attenuated inversion recovery MRI after IVMP showing diminishment of the hyperintense lesions (left panel) in the tegmentum of the pons (top left) and the dorsal medulla oblongata including the area postrema (bottom left). A sagittal spinal T2-weighted MRI revealed diminishment of the hyperintense lesions (right panel). b Visual field test scores for the left eye from the Humphrey Field Analyzer 30-2 program, at the onset of optic neuritis (left) and after additional steroid pulse and plasma exchange (right). c Coronal (left panel) and axial (right panel) contrast-enhanced MRI showing swelling and enhancement of the bilateral optic nerves. 